\title{
Inhalt 2018/1
}

\section{Zur Einführung}

STEFFEN HÖHNE, JULIA GLESNER

Wissenschaftliche Beiträge Research Articles

Die Neustrukturierung der Altersbeziehung kultureller Partizipation

Ein Langzeitvergleich bundesweiter Bevölkerungsumfragen KARL-HEINZ REUBAND

Essays und Fallstudien Essays and Case Studies

Audience development in the migratory society Insights for cultural institutions based on current research VERA ALLMANRITTER

Rezeptionszentrierung als zentraler strategischer Managementansatz:

Implikationen für Forschung, Produktentwicklung und Management ANNETTE LÖSEKE

Möglichkeiten und Grenzen kulturpolitischer Transformation am aktuellen Beispiel Thüringen TOBIAS J. KNOBLICH 
Öffentliche Kulturförderung und ihre Nebenfolgen

Die digitale Gretchenfrage Wie hast du's mit Google?

Stress in Music Managers and Artists:

Pilot study on Czech and Slovak Students KATEŘINA VAŠÍČKOVÁ, ANDREA MIKOTOVÁ, LUCIE ŠILEROVÁ

Arbeits - und Tagungsberichte Work and Conference Reports

Historische Theaterpublikumsforschung. Ein Überblick über neue Untersuchungen STEFFEN HÖHNE

Connected Audience Conference 2017, 14.-16. September 2017, Wien VERA ALLMANRITTER

Rezensionen

Reviews

Patrick S. FÖHL, Patrick GLOGNER-PILZ:

Kulturmanagement als Wissenschaft.

Grundlagen - Entwicklungen - Perspektiven.

Einführung für Studium und Praxis

TASOS ZEMBYLAS 
Gregory SHOLETTE: Delirium and Resistance:

Activist Art and the Crisis of Capitalism

JAKOB WIRTH

Christiane SCHÜRKMANN: Kunst in Arbeit.

Künstlerisches Arbeiten zwischen Praxis und Phänomen.

LETICIA LABARONNE

Thomas RENZ: Nicht-Besucherforschung.

Die Förderung kultureller Teilhabe

durch Audience Development

MANUELA BERNECKER

Michael HUBER: Musikhören im Zeitalter Web 2.0 Theoretische Grundlagen und empirische Befunde MORITZ STEINHAUER 
